\title{
Revista mídia e cotidiano
}

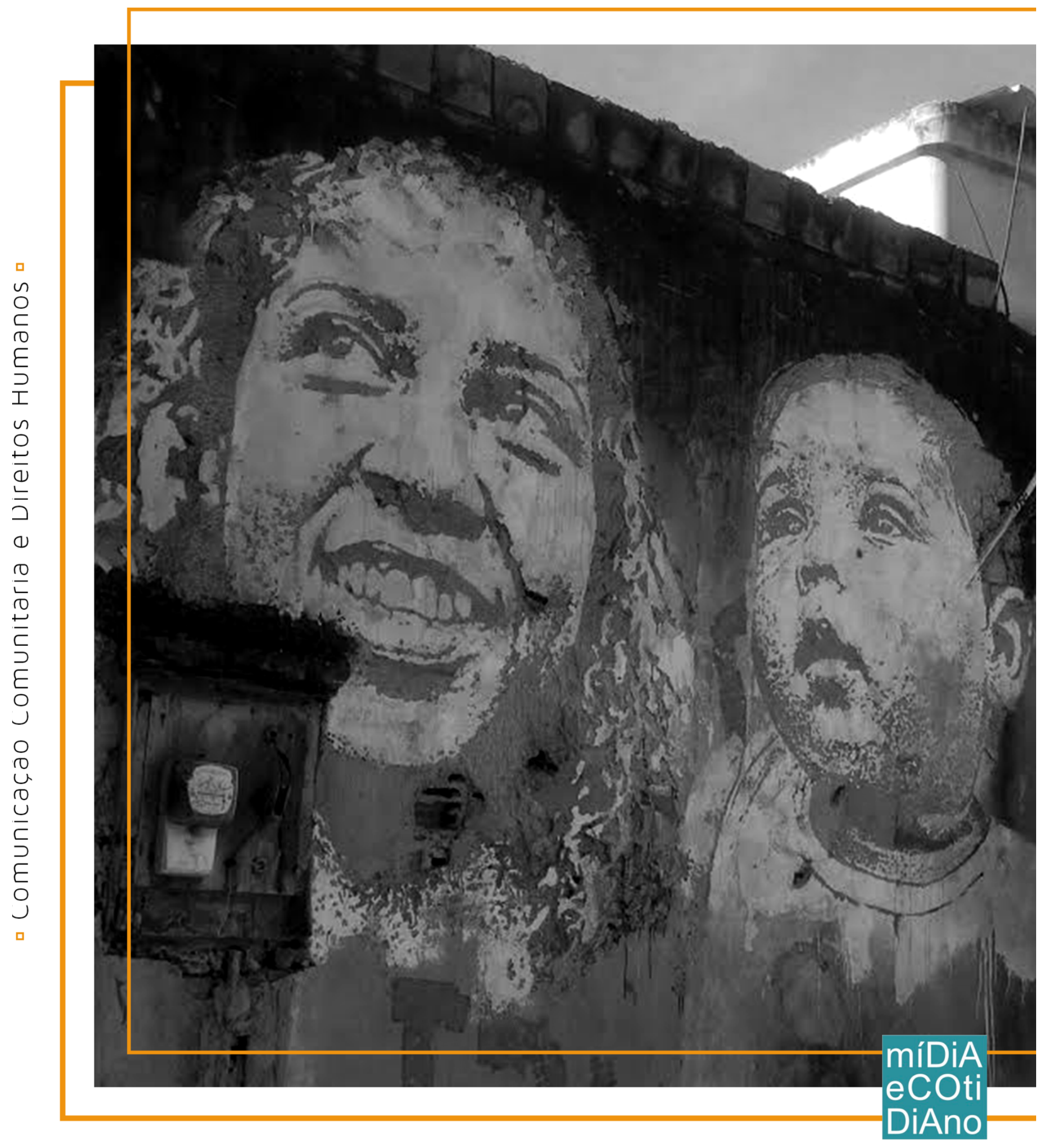

|l|| Volume 11 || número 1 || abril de 2017 ISSN 2178-602X

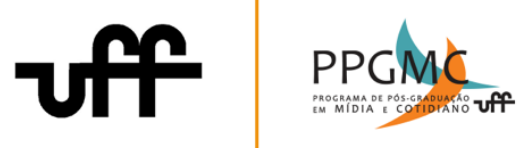




\section{míDiA \\ eCOti \\ DiAno}

\section{Sumário}

\section{Editorial}

Carla Baiense; Patrícia Saldanha

\section{Artigos}

\section{Seção Temática}

CONTRIBUICÕES DA REDE DE COMBATE AO TRABALHO

ESCRAVO NA CONSTRUÇÃO DE REPRESENTAÇÕES DA

TEMÁTICA NA MÍDIA: análise da atuação da CPT (Comissão Pastoral

da Terra) e do MHuD (Movimento Humanos Direitos)

CONTRIBUTIONS OF THE LABOR SLAVE NETWORK IN THE CONSTRUCTION OF THEMATIC REPRESENTATIONS IN THE MEDIA: analysis of the CPT (Pastoral Land Commission) and of MHuD (Human Rights Movement)

Flavia MOURA; Osmilde Augusto MIRANDA

RÁDIOS COMUNITÁRIAS E DIREITOS HUMANOS. O CASO DA

RÁDIO SERRA FM: desafios e atitudes do fazer comunicação

comunitária em um dos municípios de menor idh do país

COMMUNITY RADIOS AND HUMAN RIGHTS. THE CASE OF RADIO SERRA FM: challenges and attitudes of doing community communication in one of the municipalities with the lowest hdi in brazil

Orlando Maurício de Carvalho BERTI 
IDENTIDADE, HISTÓRIA E IMAGEM NO PROCESSO DE 46-65

CRIAÇÃO DA MARCA DO CONJUNTO PALMEIRAS DURANTE A

COPA DO MUNDO FIFA 2014

IDENTITY, HISTORY AND IMAGE IN THE PROCESS OF CREATING THE CONJUNTO PALMEIRAS BRAND DURING THE 2014 FIFA WORLD CUP

Sílvia Helena BELMINO; Emylianny Brasil DA SILVA; Luminita-Anda MANDACHE

COMUNICAÇÃO, EDUCAÇÃO, CULTURA E SUAS MEDIAÇÕES: 66-87 uma incursão no projeto Proteger é Preciso/ONG Oficina de Imagens

COMMUNICATION, EDUCATION, CULTURE AND THEIR MEDIATIONS: an experience with the project Proteger é Preciso/ONG Oficina de Imagens

Camila de ALVARENGA; José Márcio BARROS

AS REPRESENTAÇÕES SOCIAIS DA IDENTIDADE SURDA E O 88-105 DIREITO AO RECONHECIMENTO

THE SOCIAL REPRESENTATIONS OF DEAF IDENTITY AND THE RIGHT TO RECOGNITION

Márcia VIDAL NUNES; Marina GOMES PORTELA

RÁDIO POSTE DA QUADRA: uma rádio em transformação em uma 106-120 comunidade de Fortaleza (CE)

QUADRA'S LAMP POST RADIO: radio in transformation in a community in Fortaleza (CE)

Catarina Tereza Farias de OLIVEIRA; Milena de Castro RIBEIRO 


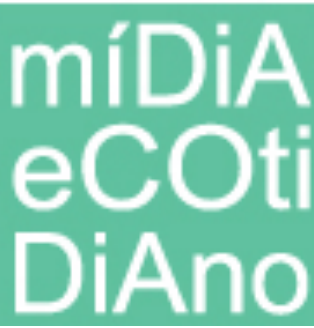

INTERVENÇÃO CIDADÃ: Nelson Mandela e a política dos direitos

121-140

humanos na África do Sul

CITIZEN INTERVENTION: Nelson Mandela and the Politics of Human

Rights in South Africa

Ingrid GOMES

Seção Livre

PRÁTICAS SITUACIONISTAS EM PROJETOS ARTÍSTICOS DE

141-155

MÍDIAS LOCATIVAS

SITUATIONIST PRACTICES IN LOCATIVE MEDIA ARTISTIC PROJECTS

Tiago Ricciardi Correa LOPES

TERRORISMO MIDIÁTICO: disseminação do medo em programa televisivo francês

156-170

NEWSWHORTHY TERRORISM EVENT: the spread of fear in French TV

Janaina Cardoso da SILVA; Christina Ferraz MUSSE

DIREITOS HUMANOS E COTIDIANO: uma visada empírica

171-189

HUMAN RIGHTS AND EVERYDAY LIFE: an empirical view

José Ricardo CUNHA 


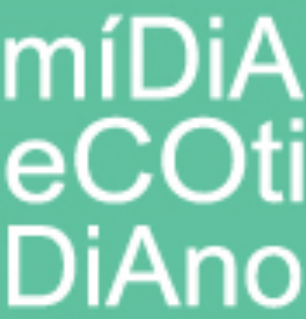

HOMOSSEXUALIDADE E ALTERIDADE: uma análise bakhtiniana

190-206 das Paradas Gays

HOMOSEXUALITY AND ALTERITY: a bakhtinian analysis of Gay Parades

João Paulo MALERBA

BOTAFOGO E O CANAL 100: documentário e o ethos de memórias no jornalismo esportivo

BOTAFOGO AND CANAL 100: documentary and the ethos of memories in sports journalism

Rafael Duarte Oliveira VENANCIO; Isabel Cristina de Oliveira GONÇALVES

\section{Resenha}

ETNOGRAFIA E CONSUMO MIDIÁTICO: expondo desafios e abordando novas soluções

ETNOGRAPHY AND MEDIA COMSUMPTION: exposing the challenges and approaching new solutions

Fernanda COSTANTINO; Juliana SOUZA 


\section{Entrevista}

O DESAFIO DE REFUNDAR A COMUNICAÇÃO COMUNITÁRIA:

227-236

Raquel Paiva indica as potencialidades e faz um balanço do campo de

estudos que consolidou no Brasil

THE CHALLENGE OF REESTABLISHING COMMUNITY

COMMUNICATION: Raquel Paiva balances and indicates the potentials of the field that consolidated Brazil

Raquel BERTOL 
A Revista Mídia e Cotidiano (ISSN 2178-602X), é uma publicação quadrimestral acessível em meio eletrônico, veiculando artigos elaborados por pesquisadores da área de Comunicação e afins.

Editada pelo Programa de Pós-Graduação em Mídia e Cotidiano (PPGMC) da Universidade Federal Fluminense (UFF), e vinculada ao Laboratório de Pesquisas Aplicadas em Mídia e Cotidiano (LaPA), a revista apresenta em suas edições artigos, resenhas, espaços para entrevistas e seções especiais, tendo como proposta ser um espaço para discussão e divulgação de trabalhos científicos relevantes para o cenário das pesquisas em Comunicação elaborados por pesquisadores doutores (mestres e alunos de pós-graduação podem enviar artigos em coautoria com orientadores doutores), fomentando a produção cultural, o debate e o diálogo na comunidade acadêmica.

A linha editorial da revista privilegia as perspectivas crítica e dialética na análise das interações midiatizadas dos sujeitos e das coletividades. Esta abordagem vai além do senso comum e da aparente banalidade da vida cotidiana, que pode ser compreendida e transformada. Em seus diversos modos de apreensão, toma como fio condutor o olhar sobre acontecimentos e dinâmicas dos processos de produção midiática em suas distintas relações com a sociedade e a cultura.

Seus artigos tratam das formas de produção de sentido e construção de identidade decorrentes da relação entre os diferentes agentes sociais e os discursos midiáticos em distintos suportes, especialmente nos campos da publicidade, do jornalismo, do audiovisual e do design. Organizam-se em torno de pesquisas sobre linguagem, discurso, retórica, representação e consumo no cotidiano das práticas e discursos midiáticos com ênfase em questões culturais, sociabilidades e estéticas.

Sumarizando, a revista pretende reunir textos contemplando condições de recepção dos discursos midiáticos, estratégias de produção de sentido e representações da memória social, voltados a compreender construções discursivas e processos midiáticos no contexto das relações de poder observáveis em diversas práticas sociais da vida cotidiana, organizando-se também em torno de pesquisas sobre os meios de comunicação hegemônicos, contra e não-hegemônicos, e sobre iniciativas contradiscursivas de transformação social. Ela enfoca as diferentes possibilidades de apropriação das tecnologias da informação e da comunicação, pela relação do jornalismo e da publicidade com a questão social, a cultura e a memória histórica, e pelas interfaces da comunicação com o local, o regional e o global.

Os objetivos gerais da Revista Mídia e Cotidiano são:

a) agregar maior projeção às pesquisas relacionadas à sua linha editorial; 
b) divulgar pesquisas empíricas ou teóricas nas temáticas relacionadas ao campo da Comunicação no Brasil e no mundo;

c) elaborar dossiês e seções temáticas a partir da seleção de trabalhos de especialistas em assuntos previamente sinalizados. 


\section{Equipe Editorial}

\section{Editores Gerais}

Prof. Dra. Andrea Medrado, Universidade Federal Fluminense - UFF (PPGMC; Dept ${ }^{\circ}$ de Comunicação / IACS), Brasil

Prof. Dr. Emmanoel Ferreira, Universidade Federal Fluminense - UFF (PPGMC; Dept $^{\mathrm{o}}$ de Estudos Culturais e Mídia / IACS), Brasil

\section{Editores da Seção Temática "Comunicação Comunitária e Direitos Humanos"}

Prof. Dra. Carla Baiense, Universidade Federal Fluminense - UFF (PPGMC; Dept ${ }^{\circ}$ de Estudos Culturais e Mídia / IACS), Brasil

Prof. Dra. Patrícia Saldanha, Universidade Federal Fluminense - UFF (PPGMC; Dept ${ }^{\circ}$ de Comunicação / IACS), Brasil

\section{Conselho Editorial}

Prof. Dr. Adilson Cabral, Universidade Federal Fluminense (PPGMC; Dept ${ }^{\mathrm{o}}$ de Comunicação; IACS/UFF), Niterói, Brasil

Prof. Dr. Alexandre Farbiarz, Universidade Federal Fluminense (PPGMC; Dept ${ }^{\circ}$ de Comunicação; IACS/UFF), Niterói, Brasil

Prof. Dra. Ana Paula Bragaglia, Universidade Federal Fluminense (PPGMC; Dept ${ }^{\circ}$ de Comunicação; IACS/UFF), Niterói, Brasil

Prof. Dra. Andrea Medrado, Universidade Federal Fluminense (PPGMC; Dept ${ }^{\circ}$ de Comunicação; IACS/UFF), Niterói, Brasil

Prof. Dra. Carla Baiense Felix, Universidade Federal Fluminense (PPGMC; Dept ${ }^{\circ}$ de Comunicação; IACS/UFF), Niterói, Brasil

Prof. Dra. Denise Tavares, Universidade Federal Fluminense (PPGMC; Dept ${ }^{\circ}$ de Comunicação; IACS/UFF), Niterói, Brasil

Prof. Dr. Emmanoel Ferreira, Universidade Federal Fluminense (PPGMC; Dept ${ }^{\mathrm{o}}$ de Estudos Culturais e Mídia; IACS/UFF), Niterói, Brasil

Prof. Dr. Guilherme Nery Atem, Universidade Federal Fluminense (PPGMC; Dept ${ }^{\circ}$ de Comunicação; IACS/UFF), Niterói, Brasil

Prof. Dr. João Batista de Abreu Junior, Universidade Federal Fluminense (PPGMC; Dept $^{\circ}$ de Comunicação; IACS/UFF), Niterói, Brasil 


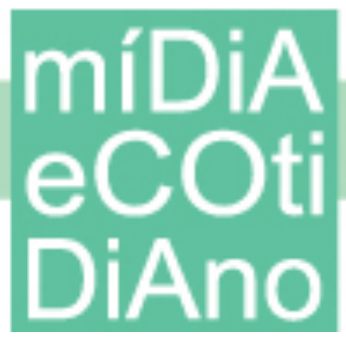

Prof. Dra. Laura Bredan, Universidade Federal Fluminense (PPGMC; Dept ${ }^{\circ}$ de Comunicação; IACS/UFF), Niterói, Brasil

Prof. Dr. Marco Schneider, Universidade Federal Fluminense (PPGMC; Dept ${ }^{0}$ de Comunicação; IACS/UFF), Niterói, Brasil

Prof. Dra. Patrícia Gonçalves Saldanha, Universidade Federal Fluminense (PPGMC; Dept $^{\circ}$ de Comunicação; IACS/UFF), Niterói, Brasil

Prof. Dra. Renata Rezende, Universidade Federal Fluminense (PPGMC; Dept ${ }^{\circ}$ de Comunicação; IACS/UFF), Niterói, Brasil

\section{Conselho Científico}

Prof. Dra. Aimée Vega-Montiel, Universidad Nacional Autónoma de México - UNAM, México

Prof. Dra. Clementina Galera Casquet, Universidad de Extremadura, Espanha

Prof. Dr. Dênis de Moraes, Universidade Federal Fluminense - UFF, Brasil

Prof. Dr. Francisco Karam, Universidade Federal de Santa Catarina - UFSC, Brasil

Prof. Dr. Gabriel Kaplún, Universidad de la República Uruguay, Uruguai

Prof. Dra. Liriam Sponholz, Universität Erfurt, Alemanha

Prof. Dra. Lucília de S. Romão, Universidade de São Paulo - USP, Brasil

Prof. Dr. Marcius Freire, Universidade Estadual de Campinas - UNICAMP, Brasil

Prof. Dr. Marcos Palácio, Universidade da Beira Interior - UBI, Portugal

Prof. Dra. Maria Aparecida Baccega, Escola Superior de Propaganda e Marketing ESPM, Brasil

Prof. Dra. Marta Martín Llaguno, Universidad de Alicante - UA, Espanha

Prof. Dr. Massimo Canevacci, Università degli Studi di Roma La Sapienza, Itália; Universidade do Estado do Rio de Janeiro - UERJ, Brasil

Prof. Dr. Muniz Sodré, Universidade Federal do Rio de Janeiro - UFRJ, Brasil

Prof. Dra. Nilda A. Jacks, Universidade Federal do Rio Grande do Sul - UFRGS, Brasil

Prof. Dra. Raquel Paiva, Universidade Federal do Rio de Janeiro - UFRJ, Brasil

\section{Conselho Consultivo do Volume 11, Número 1}

Prof. Dr. Adilson Cabral, Universidade Federal Fluminense - UFF, Brasil

Prof. Dra. Ana Enne, Universidade Federal Fluminense - UFF, Brasil

Prof. Dra. Ana Paula Bragaglia, Universidade Federal Fluminense - UFF, Brasil 
Prof. Dra. Denise Tavares, Universidade Federal Fluminense - UFF, Brasil

Prof. Dr. João Batista de Abreu Junior, Universidade Federal Fluminense - UFF, Brasil

Prof. Dr. Juciano Lacerda, Universidade Federal do Rio Grande do Norte - UFRN, Brasil

Prof. Dra. Laura Bedran, Universidade Federal Fluminense - UFF, Brasil

Prof. Dr. Leonardo de Marchi, Universidade do Estado do Rio de Janeiro - UERJ, Brasil

Prof. Dr. Leonel Aguiar, Pontifícia Universidade Católica do Rio de Janeiro - PUC-RJ, Brasil

Prof. Dra. Pamela Craveiro Uchoa, Universidade Federal Fluminense - UFF, Brasil

Prof. Dra. Renata Rezende, Universidade Federal Fluminense - UFF, Brasil

Prof. Dr. Rozinaldo Miani, Universidade Estadual de Londrina - UEL, Brasil

\section{Capa}

\section{Projeto Gráfico e Design}

Vitor Silva Chagas, aluno de Comunicação Social/Publicidade, Universidade Federal Fluminense - UFF, Brasil

\section{Foto de Capa}

Prof. Andrea Medrado, PPGMC - UFF

Data da publicação: 17 de maio de 2017 